\title{
Optimizing Transition arc between Blot Head and Blot Bar to Obtain Minimum Stress Concentration
}

\author{
Yazhou Xu \\ Mechanical Engineering College, \\ Yangzhou University \\ Yangzhou, P. R. China \\ yazhouxu@163.com
}

\author{
Tian Shuai \\ Mechanical Engineering College, \\ Yangzhou University \\ Yangzhou, P. R. China \\ metianshuai@163.com
}

\author{
Zhixue $\mathrm{Wu}$ \\ Mechanical Engineering College, \\ Yangzhou University \\ Yangzhou, P. R. China \\ zhixue_wu@yahoo.com
}

\begin{abstract}
An effective parameterization method is proposed to optimize transition arc's shape between blot head and blot bar for minimum stress concentration. This method adopts super circular curve equation to describe transition arc's shape, then selecting equation's parameters as the design variables. Thus it becomes very easy to describe and obtain optimal transition arc's shape just making use of the optimization module provided in a commercial finite element package due to the less number of design variables. Finally, an example of hexagonal bolt M64 is presented and discussed to demonstrate the accuracy and the practical of the proposed shape optimization method.
\end{abstract}

Keywords-bolt;super circular;Stress concentration;optimization design;

\section{INTRODUCTION}

In the engineering, bolt is one of the most popular fitings and the strength of the bolt connection is also mainly depending on them. In terms of the failure property, about $90 \%$ of the failure belong to fatigue damage, and about $15 \%$ of these come from transition arc between blot head and blot bar. It will have extremely important realistic meanings for improving the strength of the bolt connection if we could find a way to reduce the Stress concentration of transition arc between blot head and blot bar. Some method such as increasing the fillet or adding unloading structures are frequently used to resolve the problem of how to reduce the Stress concentration of transition arc between blot head and blot bar. The transition arc radius $\mathrm{R}$ is usually given by conventional formula, therefore, this method tends to can't reduce the effect of stress concentration effectively or maybe can't get a optimal transition curve between blot head and blot bar.

There is no doubt that choosing a suitable optimization method is very important, however, it is usually very difficult to get an optimal boundary due to the highly non-linear between element stiffness matrix or other factors and design variables.

Finite element based methods could be described directly by the node coordinates, but this method can cause the design variables increased. For this problem, here must take some special methods such as all kinds of the gradientless optimization methods [7-9] to solve it. And another, even

The present work was supported by the National Natural Science Foundation of China under Grant No. 51075354. more serious problem is that this method can't guarantee the smoothness of the design boundary so the boundary maybe looks like a sawtooth. In order to get a smooth continuous boundary, design boundary of the structure was usually defined by spline curves passing through a number of control points [1-6]. It is certainly that this method could describe most of the boundary curve shape. Zhixue [14] has ever simulated the plants' growing process to get the minimum stress concentration of a hole by using this method, and a good result was reported. However, the optimization efficiency will reduce greatly and also not suitable for optimization modules which has been incorporated into commercial finite element packages due to the precision of this method depends on the number of control points, all of this make the commonality and the ease of use to be bad. Literatures [10-13] describe the design boundary with one type of the analytical equation. Compared with the two methods mentioned above, it is clearly that this method is more beneficial since it could define the shape of mode using just a few variables and obtain a smooth boundary. But perhaps the biggest complain of this method is that user maybe can't find a proper parametric expression or could only find the local optimal solution. Although this method has some disadvantage, it was still applied to many structural optimization problems because of its simplicity and effectiveness.

If we can find out a suitable analytic equation, it will ecstatically improve the efficiency and reduce the optimal difficulty. besides that, one can use the optimized software's optimization module easily. Base on this point, we adopt super circular equation to optimize transition arc's shape and verify its accuracy and practicality.

\section{Parameterization Of The Bolts}

Here we just select the hexagonal bolt M64 as our research object. The task is to change the Transition arc's shape of bolts which has been increasing the fillet or adding unloading structures for minimum stress concentration. The geometries are shown in Fig.1. and Fig.2. All of the relevant parameters were presented by GB/T5728-2000 and the length of the bolt bar is $220 \mathrm{~mm}$. We select $35 \mathrm{CrMnSiA}$ as the bolt material. For this analyses liner elastic constitutive model is used with Yong's modulus of $206 \mathrm{GPa}$ and poisson's ration of 0.28 . 


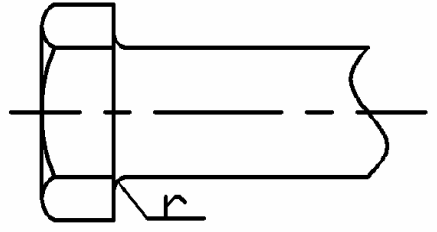

Fig.1 The bolts which has been increasing the fillet

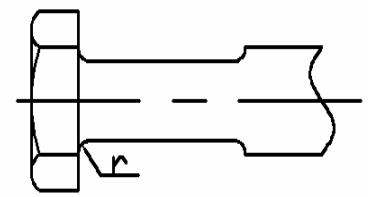

Fig. 2 The bolts which has been adding unloading structures

In practice, the Constraints and the working load are all symmetrically along the axis, so all of the stress v the strain and the displacement are symmetrical distribution along the axis, therefore it belongs to the space axisymmetric problems. Such problems can be simplified as a quasi two-dimensional planar problem to deal with. It is important to notice that the finite element model must be located in XOY plane of the whole Cartesian coordinate system and the symmetric axis must be $\mathrm{Y}$ axis and at the same time all coordinates of the keypoints have to meet the condition of $X \geq 0$ when one uses ANSYS to solve such issues. In addition, as the threaded section is distant from the transition arc between blot head and blot bar and they have no relation with this paper so the threaded section can be neglected.

The shape of the transition arc between blot head and blot bar is parameterized by the double exponent super circular

$$
\left(\frac{x}{R}\right)^{\eta_{1}}+\left(\frac{y}{R}\right)^{\eta_{2}}=1
$$

In rectangular coordinates $(\mathrm{x}, \mathrm{y})$. Where power $\eta_{1}$ and $\eta_{2}$ are the two parameters that control the shape of the design boundary and parameter $\mathrm{R}$ is the radius of transition arc. In special circumstances, the super circular will be transformed into a circular when $\eta_{1}=\eta_{2}=2$. So circular can be regarded as a special kind of super circular. The double exponent super circular will become the single exponent super circular while $\eta_{1}=\eta_{2}=\eta$. Compare to the graph of double exponent super circular, the single exponent super circular must be symmetric about the line $y=x$. this will greatly limit the flexibility of the curve. To obtain a better result, we use the double exponent super circular to describe the transition arc between blot head and blot bar.

In this paper, all the finite element analyses are carried out using software package ANSYS 14.0. The finite meshes are crated with eight-node axisymmetric element. Finite element mesh size is set to be $2 \mathrm{~mm}$. To reduce the impact of element size at the highly level, In this paper the meshes near the transition arc are refined 4 times. The boundary condition is that the displacement constraint of bolt's axis point $\mathrm{X}$ direction in 0 and the lower edge of the bolt head $\mathrm{Y}$ direction in 0 , then a uniform tension stress of $1 \mathrm{MPa}$ is applied along y direction at the other edge of bolt.

\section{Optimized Results And Discussions}

\section{A. Transition Arc's Stress Distribution Of The Initial model}

For ease of comparison studies, the stress distribution of the non-optimized model's transition arc between blot head and blot bar has been solved by the finite element method. Then on this basis, the Stress concentration factor has been calculated. Here the Stress concentration factor is defined by the following formula:

$$
\alpha_{\sigma}=\frac{\sigma_{\max }}{\sigma}
$$

Where $\sigma_{\max }$ is the maximum stress in the concentration sources. And $\sigma$ is the nominal stress which is calculated by the simplified formula of materials mechanics. The initial model's transition arc radius $\mathrm{R}$ is $12.8 \mathrm{~mm}$. Fig3 and Fig4 show the Von-Mises stress contours of the two initial model's transition arc. The maximum Von-Mises stress is 907.775 $\mathrm{MPa}$ and $952.389 \mathrm{MPa}$ respectively. The Stress concentration factor is 9.08 and 9.52 based on the formula 2 .

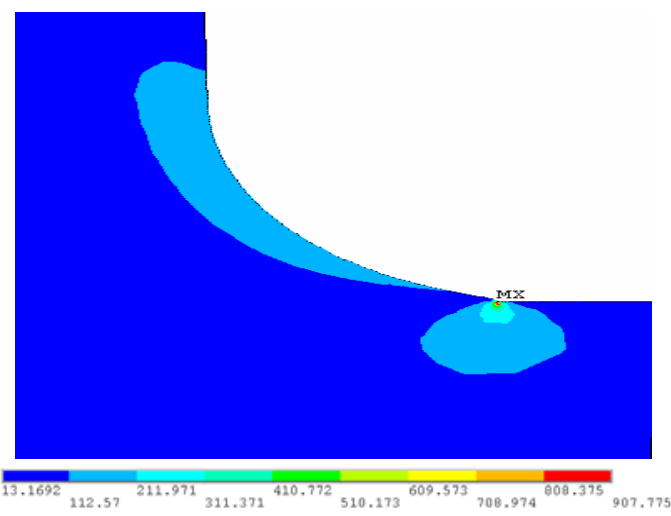

Fig.3. The Von-Mises stress contours of the bolts which has been increasing the fillet

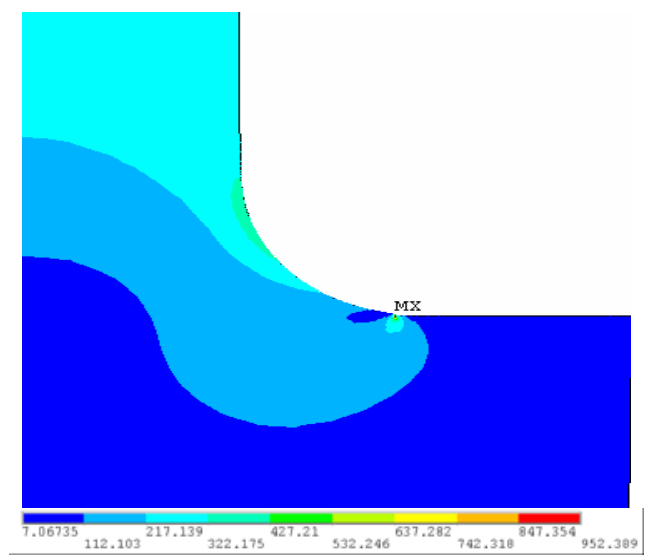

Fig.4. The Von-Mises stress contours of the bolts which has been adding unloading structures 
TABLE I. The Results Of Optimization

\begin{tabular}{|c|c|c|c|c|c|}
\hline \multirow{2}{*}{$\begin{array}{c}\text { optimization } \\
\text { models }\end{array}$} & \multicolumn{2}{|c|}{$\begin{array}{c}\text { stress } \\
\text { concentration } \\
\text { factor }\end{array}$} & \multicolumn{2}{|c|}{$\begin{array}{c}\text { Von-mises stress } \\
\text { (MPa) }\end{array}$} & \multirow{2}{*}{ parameters } \\
\cline { 2 - 5 } & $\begin{array}{c}\text { Before } \\
\text { optimi } \\
\text {-zation }\end{array}$ & $\begin{array}{c}\text { After } \\
\text { optimi } \\
\text {-zation }\end{array}$ & $\begin{array}{c}\text { Before } \\
\text { optimi } \\
\text {-zation }\end{array}$ & $\begin{array}{c}\text { After } \\
\text { optimi } \\
\text {-zation }\end{array}$ & \\
\hline $\begin{array}{c}\text { the bolts which } \\
\text { has been } \\
\text { increasing the } \\
\text { fillet }\end{array}$ & 9.08 & 5.35 & $\begin{array}{c}907.77 \\
5\end{array}$ & $\begin{array}{c}535.22 \\
5\end{array}$ & $\eta_{2}=4.183$ \\
\hline $\begin{array}{c}\text { the bolts which } \\
\text { has been } \\
\text { adding } \\
\text { unloading } \\
\text { structures }\end{array}$ & 9.52 & 7.21 & 952.38 & 720.75 & $\eta_{2}=1.1$ \\
\hline
\end{tabular}

\section{B. Optimized Results By Super Circular}

In this paper, to improve the speed and the solution precision while avoiding local convergence, we use the zeroorder optimization method to get an approximate optimal result and then use the first-order optimization method to get the best result. The power $\eta_{1}$ and $\eta_{2}$ are selected as the design variables and transition arc's maximum Von-Mises stress are selected as the objective function. Table 1 shows the results of optimization.

As expected, Compared with the results of before optimization, it can be seen that the Von-mises stress of after optimization have declined much. And the results of the bolts which has been increasing the fillet has more advantage than the bolts which has been adding unloading structures.

Fig.5 and Fig. 6 show the von-mises stress contour of the models which have been optimized. Fig.7 and Fig.8 show the boundary curves before and after optimization is made.

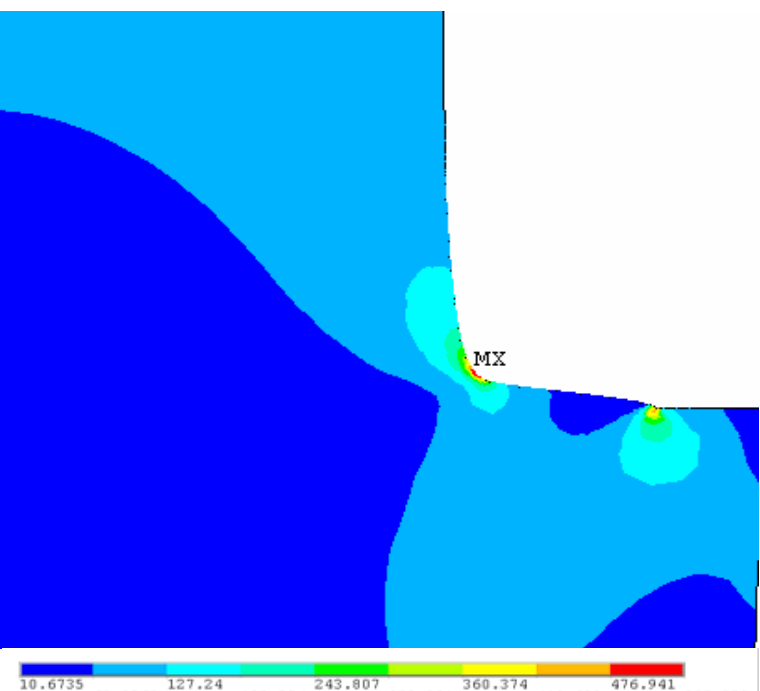

Fig.5. The von-mises stress contour of the after optimization bolts which has been increasing the fillet

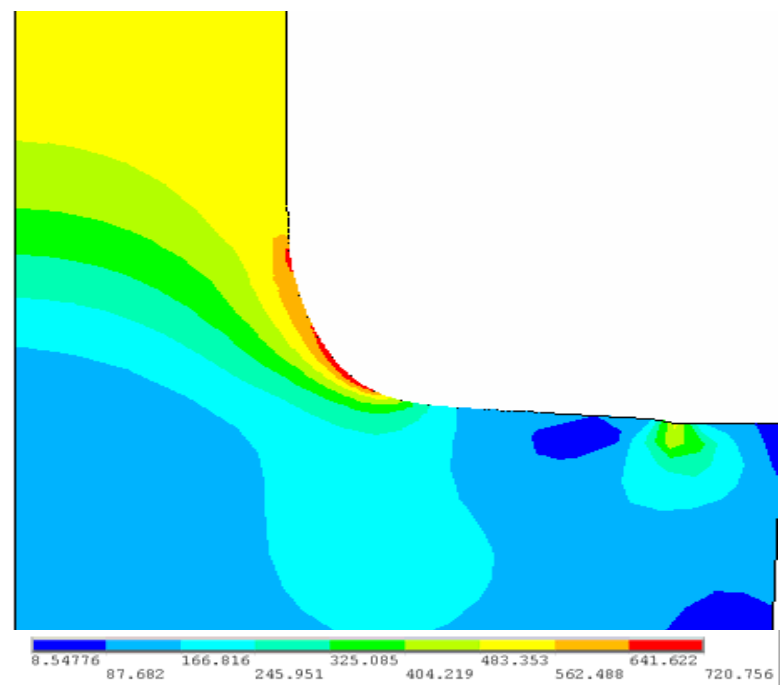

Fig.6. The von-mises stress contour of the after optimization bolts which has been adding unloading structures

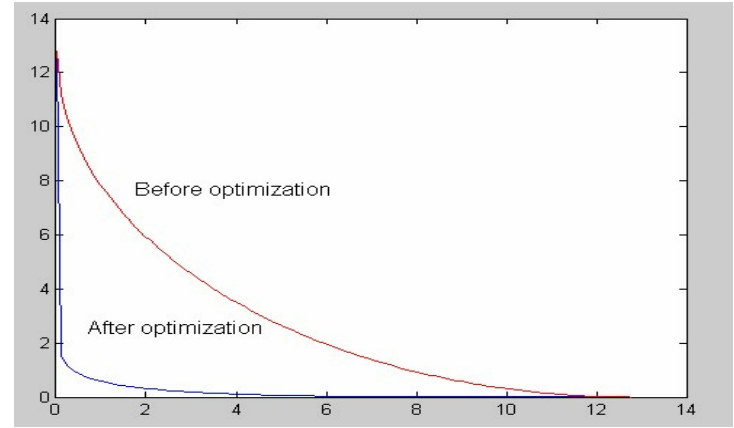

Fig.7. The boundary curves of the bolts which has been increasing the fillet

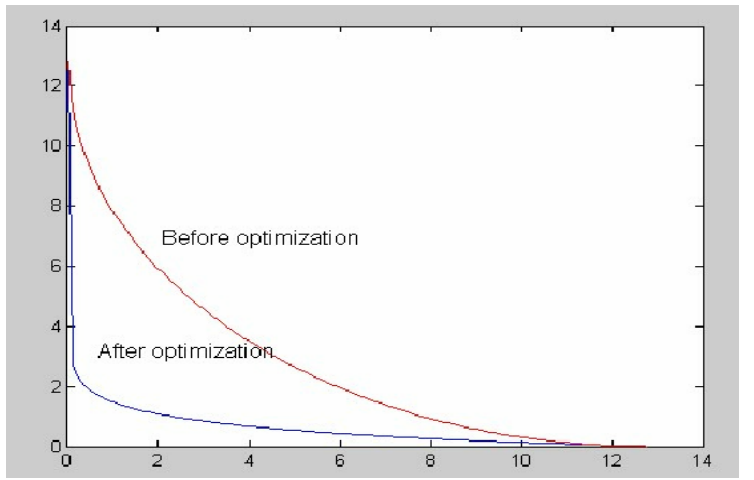

Fig.8.The boundary curves of the bolts which has been adding unloading structures

Thus, the stress concentration can be significantly reduced by using the double exponent super circular to describe transition arc between blot head and blot bar.

\section{Conclusions}

The purpose of this paper is to obtain minimum stress concentration by the double exponent super circular with finite element method. It has been shown that, applying the super circular curve equation to describe the shape of transition arc 
between blot head and blot bar, we can achieve a good result while guaranteeing the transition arc to be continuous and smooth. The number of the design variables is reduced by this method so it is easy and convenient to use in reality.

\section{REFERENCES}

[1] Zhixue W. An efficient approach for shape optimization of components [J]. International Journal of mechanical sciences, 2005, 47(10):15951610 .

[2] Sonmez F O. Shape optimization of 2D structures using simulated annealing [J]. Comput. Methods Appl. Mech. Engrg. 2007, 196: 32793299.

[3] Annicchiarico W, Cerrolaza M. Structural shape optimization 3D finiteelement models based on genetic algorithms and geometric modeling [J]. Finite Elem. Anal. Des. 2001, 37:403-415.

[4] Schnack E, Weikl W. Shape optimization under fatigue using continuum damage mechanics [J]. Comput.-Aided Des. 2002,34:929-938.

[5] Schmid F, Hirschen K, Meynen S, Schafer M. An enhanced approach for shape optimization using an adaptive algorithm [J]. Finite Elem. Anal. Des. 2005, 41:521-543.

[6] Wilczyński B. Shape optimisation for stress reduction around single and interacting notches based on the fictitious stress method [J]. Engrg. Anal. Bound. Elem. 1997, 19:117-128.
[7] Heller M, Kaye R, Rose L R. A gradientless finite element procedure for shape optimization [J]. The Journal of Strain Analysis for Engineering Design. 1999, 34:323-336.

[8] Waldman W, Heller M, Chen G X. Optimal free-form shapes for shoulder fillets in flat plates under tension and bending [J]. Int. J. Fatigue. 2001, 23:185-190.

[9] McDonald M, Heller M. Robust shape optimization of notches for fatigue-life extension [J]. Struct. Multidisc. Optim. 2000, 19:169-182.

[10] Phan A-V, Phan T-N. A structural shape optimization system using the two-dimensional boundary contour method [J], Arch. Appl. Mech. 1999, 69:481-489.

[11] Pedersen P. On optimal shapes in materials and structures [J]. Struct Multidisc Optim. 2000, 19:169-182

[12] Pedersen N L.Optimization of holes in plates for control of eigenfrequencies [J]. Struct Multidisc Optim. 2004, 28:1-10

[13] Pedersen P. Design study of hole positions and hole shapes for crack tip stress releasing [J]. Struct Multidisc Optim. 2004, 28:243-251

[14] Zhixue w. Bio-inspired optimization method for component design [J]. China Mechanical Engineering,2005,16(10):869-873 